\title{
Reflets
}

Revue d'intervention sociale et communautaire

\section{Maintien de l'engagement paternel après la rupture : point de vue de pères et de mères en contexte de pauvreté}

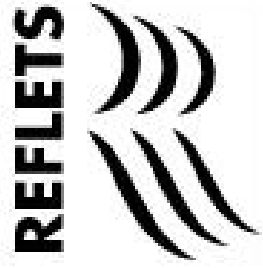

\section{Gilles Tremblay et Francine Allard}

Volume 15, numéro 1, 2009

Paternités : enjeux et perspectives (Première partie)

URI : https://id.erudit.org/iderudit/029590ar

DOI : https://doi.org/10.7202/029590ar

Aller au sommaire du numéro

Éditeur(s)

Reflets : Revue d'intervention sociale et communautaire

ISSN

1203-4576 (imprimé)

1712-8498 (numérique)

Découvrir la revue

\section{Citer cet article}

Tremblay, G. \& Allard, F. (2009). Maintien de l'engagement paternel après la rupture : point de vue de pères et de mères en contexte de pauvreté. Reflets, 15(1), 120-142. https://doi.org/10.7202/029590ar

\section{Résumé de l'article}

Depuis les dernières décennies, les ruptures d'unions conjugales se sont multipliées au Canada et au Québec, affectant maintenant $53 \%$ des ménages (Duchesne, 2006). Ainsi, près de trois hommes sur cinq (56 \%) se retrouvent dans un rôle de père gardien après la rupture (Duchesne, 2006). En dépit de changements législatifs et normatifs visant à favoriser un partage des responsabilités parentales après un divorce, la garde des enfants confiée à la mère demeure encore l'arrangement le plus courant. Les mères deviennent ainsi les principales, et souvent uniques, responsables des enfants, alors que la plupart des pères passent d'un rapport continu, régulier et intense avec leurs enfants, à une relation discontinue souvent imposée par la cour (Quéniart et Fournier, 1996).

Cet article explore le phénomène du maintien de l'engagement du père après une rupture conjugale dans un contexte de pauvreté. Après avoir situé la problématique, les résultats de deux études qualitatives sur ce sujet sont présentés (Allard, Bourret et Tremblay, 2004; 2005), l'une à partir des propos de pères qui se disent encore engagés envers leurs enfants après la rupture, l'autre à partir de la parole de mères qui considèrent leur ex-conjoint encore impliqué auprès de leurs enfants. Les participants de ces deux études n'étaient pas appariés. Par la suite, les résultats sont confrontés afin de mettre en évidence les convergences et les divergences dans les points de vue des pères et des mères. Enfin, l'article conclut avec des éléments susceptibles d'inspirer les intervenants. Rappelons que la valorisation de l'engagement paternel après la rupture conjugale des parents vise à réduire le risque que ces enfants se retrouvent doublement pauvres : pauvres économiquement et " pauvres " (privés) de père (Allard et Binet, 2002; Marsiglio et Cohan, 2000).
Tous droits réservés @ $@$ Reflets : revue d'intervention sociale et communautaire, 2009
Ce document est protégé par la loi sur le droit d'auteur. L'utilisation des services d'Érudit (y compris la reproduction) est assujettie à sa politique d'utilisation que vous pouvez consulter en ligne. 


\section{Maintien de l'engagement paternel après la rupture : point de vue de pères et de mères en contexte de pauvreté}

Gilles Tremblay

Professeur, École de service social, Université Laval

$e t$

Francine Allard

Médecin, Direction de santé publique de la Capitale-Nationale

Depuis les dernières décennies, les ruptures d'unions conjugales se sont multipliées au Canada et au Québec, affectant maintenant $53 \%$ des ménages (Duchesne, 2006). Ainsi, près de trois hommes sur cinq $(56 \%)$ se retrouvent dans un rôle de père gardien après la rupture (Duchesne, 2006). En dépit de changements législatifs et normatifs visant à favoriser un partage des responsabilités parentales après un divorce, la garde des enfants confiée à la mère demeure encore l'arrangement le plus courant. Les mères deviennent ainsi les principales, et souvent uniques, responsables des enfants, alors que la plupart des pères passent d'un rapport continu, régulier et intense avec leurs enfants, à une relation discontinue souvent imposée par la cour (Quéniart et Fournier, 1996).

Cet article explore le phénomène du maintien de l'engagement du père après une rupture conjugale dans un contexte de pauvreté. Après avoir situé la problématique, les résultats de deux études qualitatives sur ce sujet sont présentés (Allard, Bourret et Tremblay, 2004;2005), l'une à partir des propos de pères qui se disent encore 
engagés envers leurs enfants après la rupture, l'autre à partir de la parole de mères qui considèrent leur ex-conjoint encore impliqué auprès de leurs enfants. Les participants de ces deux études n'étaient pas appariés. Par la suite, les résultats sont confrontés afin de mettre en évidence les convergences et les divergences dans les points de vue des pères et des mères. Enfin, l'article conclut avec des éléments susceptibles d'inspirer les intervenants. Rappelons que la valorisation de l'engagement paternel après la rupture conjugale des parents vise à réduire le risque que ces enfants se retrouvent doublement pauvres : pauvres économiquement et "pauvres » (privés) de père (Allard et Binet, 2002; Marsiglio et Cohan, 2000).

\section{Que savons-nous du maintien de l'engagement paternel après la rupture en situation de pauvreté?}

Selon Gaudet, Devault et Bouchard (2005), un nombre important de pères sont absents de la vie de leur enfant quelques années après un divorce ou une séparation. Plusieurs chercheurs ont étudié la trajectoire des pères après la rupture (Dulac, 1998; Kruk, 1993), dont Hetherington et Kelly (2002) qui proposent que l'engagement paternel après la rupture se décline selon trois principaux parcours : a) les pères qui étaient engagés avant la rupture et le sont après ou ceux non impliqués avant, et qui ne le sont toujours pas après;2) les pères qui prennent conscience de l'importance des enfants dans leur vie après la séparation et qui décident alors de s'engager; 3 ) et enfin, les pères démotivés par le divorce qui abandonnent leurs enfants, parce qu'insatisfaits de la discontinuité de leur relation avec eux ou épuisés de se battre contre la résistance de la mère à leur engagement. Quels facteurs facilitent le maintien de l'engagement paternel après la rupture? Gaudet et collab. (2005) identifient : le degré d'implication du père envers ses enfants avant la rupture; l'intensité avec laquelle il s'identifie au rôle parental; le soutien social dont il bénéficie; la qualité de la relation avec son ex-conjointe, la mère des enfants. 
"La précarité

économique est

en soi un facteur susceptible de fragiliser l'engagement paternel, voire de contribuer au désengagement. Certes la barrière financière de la pauvreté constitue un frein à l'exercice du rôle de père après la rupture. Citons l'exemple du manque d'argent pour utiliser un moyen de transport nécessaire pour aller visiter son enfant..."
D'autres variables ont également été étudiées soit : le sentiment de compétence parentale (Hetherington et Stanley-Hagan, 2002); la pression exercée sur le père par l'entourage (Ihinger-Tallman, Pasley et Buehler, 1995); et enfin, le sentiment du père d'avoir du pouvoir sur les décisions relatives aux activités et au bien-être des enfants (Madden-Derdich et Leonard, 2000). À l'inverse, Hetherington et Stanley-Hagan (2002) ont constaté que les pères séparés qui se retrouvent dans une situation où ils craignent de perdre du pouvoir sur les décisions se rapportant à leurs enfants, auraient davantage tendance à être insatisfaits dans leur rôle de parent et, par la suite, à se désengager.

Comment l'engagement paternel après la rupture se conjuguet-il à la pauvreté? La précarité économique est en soi un facteur susceptible de fragiliser l'engagement paternel, voire de contribuer au désengagement. Certes la barrière financière de la pauvreté constitue un frein à l'exercice du rôle de père après la rupture. Citons l'exemple du manque d'argent pour utiliser un moyen de transport nécessaire pour aller visiter son enfant (Amato et Solewski, 2004). Rappelons que la distance géographique est rapportée par les pères non gardiens pour expliquer la diminution de la fréquence des visites (Quéniart, 2004). Ce qui donne, selon Amato et Sobolewski (2004), à la distance géographique le statut de variable clé pour expliquer la fréquence des contacts entre le père non résident et son enfant. Cela a d'autant plus d'effets négatifs que ces pères utilisent peu le courrier ou encore le téléphone pour établir des contacts avec leurs enfants (LeBourdais, Judy et Marcil-Gratton, 2000).

Mais la pauvreté a sur les pères des effets néfastes beaucoup plus intenses que chez les mères. En fait, devant une perte d'emploi (Fagan, 2000), ou devant une situation de pauvreté (Elder, Nguyen et Caspi, 1985), les pères éprouvent des réactions de stress plus sévères que celles des mères et leurs répercussions risquent de miner la qualité de leur relation avec leur enfant. Comment expliquer cette situation? Une hypothèse serait que la pauvreté économique touche de plein fouet à la composante centrale du rôle paternel traditionnel : celle de pourvoyeur (Mosley et Thomson, 1995). L'étude qualitative notoire de Liebow (1967) 
décrit justement le parcours d'hommes vivant en grande pauvreté qui en viennent progressivement à se dissocier eux-mêmes de leur famille, en partie parce qu'ils ne peuvent plus affronter le rappel quotidien de leur incapacité à pourvoir aux besoins de leurs enfants, ce qui les conduit à un désengagement ultime. Les mères aussi peuvent contribuer à la mise à l'écart du père pauvre. Une autre étude plus récente en contexte de pauvreté (Coley et Morris, 2002) rapportait cette fois que des mères avaient tendance à bloquer l'accès au père lorsqu'il était incapable de contribuer financièrement aux besoins de l'enfant. Chez les hommes défavorisés économiquement, d'autres facteurs peuvent accroître le caractère périphérique de leur rôle paternel après une rupture. Citons l'exemple des tribunaux qui peuvent considérer la pauvreté économique du père comme un élément négatif, comme un obstacle à l'octroi de la garde partagée, à la différence de la mère pour qui ce motif n'est jamais invoqué (Joyal et collab., 2003). Quel regard des pères et des mères pauvres portent-ils sur le maintien de l'engagement paternel après la rupture?

\section{Les deux études qualitatives réalisées}

\section{Concept d'engagement paternel}

Les deux études reposent sur le concept d'engagement paternel décrit par Lamb, Pleck et Levine (1986) et enrichi par l'équipe de ProsPère (2004). C'est-à-dire, l'engagement paternel peut se manifester par l'une ou l'autre des dimensions suivantes : un père responsable : une prise en charge des tâches indirectes et des responsabilités relatives à l'enfant; un père affectueux : une disponibilité ainsi qu'un soutien affectif et cognitif; un père qui prend soin : une participation active aux différents soins physiques de l'enfant; un père en interaction : des interactions père/enfant significatives; un père pourvoyeur : une contribution aux soutiens financier et matériel; un père évocateur : des évocations spontanées qui révèlent l'importance de la relation avec son enfant ou le 
plaisir qu'elle suscite chez lui. D'autres auteurs ont proposé d'autres dimensions dites "indirectes ": un père qui donne accès à différents réseaux sociaux; un père qui soutient sa conjointe ou son ex-conjointe; et un père évoqué, c'est-à-dire, l'image que l'enfant développe de son père à partir de la médiation qu'en fait la mère et les autres membres de son réseau social (Lanoue et Cloutier, 1996). Ces concepts ont été utilisés comme une référence permettant d'alimenter l'interprétation et la discussion des résultats, et non dans le but d'enfermer les propos des participants dans des a priori normatifs.

\section{Participants et objectifs}

Dans la première étude, quinze pères séparés pauvres et qui se disaient engagés envers leurs enfants ont été interrogés. L'objectif était de comprendre la trajectoire de leur engagement paternel après la rupture conjugale, les stratégies qu'ils avaient utilisées pour rester engagés dans un contexte de pauvreté et enfin, de dégager les éléments qui semblaient avoir contribué à préserver leur engagement paternel. La deuxième étude a recueilli et analysé les propos de quinze mères séparées pauvres et qui considéraient leur ex-conjoint impliqué auprès de leur enfant. Ce groupe n'était pas apparié avec le précédent. Cette étude visait à comprendre comment les mères se représentaient l'engagement paternel après la rupture, de saisir par quel processus se précise l'orientation de leur influence sur l'implication paternelle après la séparation et enfin, par quelles stratégies celle-ci s'exerce sur le maintien de l'engagement paternel.

Les pères et les mères étaient séparés depuis au moins six mois, avaient au moins un enfant d'âge préscolaire et vivaient en situation de pauvreté. Les caractéristiques retenues pour définir la situation de pauvreté étaient celles des clientèles des programmes de santé publique destinés aux parents vulnérables d'enfants d'âge préscolaire (INSPQ, 2000), soit : avoir recours à l'assistance-emploi et détenir un niveau de scolarité équivalant au secondaire $\mathrm{V}$ ou moins. Les principaux agents de recrutement dans les deux cas ont été les organismes communautaires et de services aux familles dans les quartiers centraux de la ville de Québec. 
Les pères de l'échantillon étaient âgés de 35 à 42 ans, la moitié d'entre eux n'avaient pas terminé leurs études secondaires et plus de la moitié vivaient de l'assistance-emploi. Au moment de l'entretien, six partageaient la garde avec leur ex-conjointe, quatre avaient la garde complète de leurs enfants; pour trois pères, la garde était assumée par la mère et dans deux cas, les modalités n'étaient pas encore définies. L'échantillon des mères avait entre 25 et 34 ans. La plupart avaient poursuivi des études de niveau secondaire, sans avoir toutes leurs diplômes. Toutes vivaient de l'assistance-emploi. Dix assumaient la garde des enfants, quatre la partageaient avec leur ex-conjoint et un père assumait la garde de ses enfants.

\section{Méthodologie}

Les entrevues semi-structurées ont été tenues le plus souvent au domicile des participants. Les propos ont été enregistrés, retranscrits et analysés avec l'aide du logiciel NVivo 2.0. L'analyse visait notamment à faire ressortir les éléments divergents et convergents des catégories issues du canevas d'entrevue ainsi que d'autres thèmes émergents (Huberman et Miles, 1991). Les résultats de ces études ne visent aucune généralisation. Ces deux recherches ont été approuvées par un comité d'éthique. Parmi les biais, citons le mode de sélection des participants des deux études. Étant invités à parler de leur engagement parental ou de celui de leur ex-conjoint ou ex-conjointe, un tel critère a pu amener des mères et des pères, à surestimer les bons coups, soit par dignité, soit par désir d'approbation sociale. L'engagement paternel après la rupture dont parlent les participants est-il en continuité avec l'engagement avant la rupture ou plutôt du type "motivés du divorce "? Nous l'ignorons. Certes, ces critères ont exclu d'emblée les pères non impliqués ou les "démotivés du divorce " (Hetherington et Kelley, 2002). Pour la majorité, le fait d'avoir accepté de participer à l'étude peut attester une volonté, sinon un intérêt, d'aborder cette question. L'offre d'une compensation financière annoncée au moment de la sélection a pu également constituer un biais de sélection. Les conditions dans lesquelles se déroulait l'entrevue ont pu interférer sur les résultats. Enfin, les 
participantes et les participants étaient informés que les chercheurs étaient liés par la Loi sur la protection de la jeunesse. Est-ce que cela a pu les amener à taire certains événements ou sentiments? Nous ne le savons pas, mais constatons toutefois qu'ils et elles ont abordé avec confiance des sujets délicats. Les conditions des entrevues, loin d'avoir eu comme effet d'en restreindre le contenu, semblent au contraire l'avoir alimenté.

\section{Le point de vue des pères}

Après avoir traversé, dans les premiers temps suivant la rupture, ce que Dulac (1995) a appelé le "trou noir ", plusieurs des pères interrogés s'éveillent et reprennent la maitrise de leur vie. Pour regagner leur place auprès de leur enfant, ils livrent un combat autant dans l'espace public que privé. Avant d'amorcer les démarches officielles, souvent juridiques, en vue du partage des responsabilités parentales, certains ont dû effectuer des requêtes pour faire reconnaître légalement leur paternité. Affirmer la légitimité de leur rôle dans l'espace public et obtenir leur autonomie parentale a représenté un défi tel que quelques pères ont failli "décrocher ". Néanmoins, la majorité des participants rapportent le soutien de leurs proches comme un élément favorable au maintien de leur engagement paternel. Ces pères rapportent avoir redéfini leur nouveau rôle parental aussi dans l'espace privé. Pour la majorité, ils disent être capables de s'occuper de leur enfant; ils se distinguent de la mère et retrouvent leur façon personnelle d'être parent. Ils constatent que la qualité de la relation qu'ils ont développée avec leur enfant après la rupture conjugale compense les effets néfastes de la discontinuité qu'ils avaient appréhendés. Ils décrivent en effet une relation de qualité avec l'enfant, laquelle présente plusieurs des caractéristiques reconnues comme étant bénéfiques au développement de celui-ci et susceptibles de favoriser son adaptation à la rupture. Le fait d'avoir à assumer seuls le rôle de parent à part entière, quel que soit le mode de garde, force en quelque sorte ces pères à élargir les dimensions de leur engagement. Engagement qui, en fait, correspond au concept défini par Lamb (1986) et regroupe l'ensemble des dimensions proposées par ProsPère (2004). Les résultats témoignent en outre 
"Malgré leurs

faibles revenus, ces pères font tout pour assurer le bien-être et la sécurité de leur enfant afin que celui-ci ne manque de rien, quitte à demander de l'aide de leur famille ou à avoir recours à des ressources dans la communauté. La pauvreté économique ne semble toutefois pas entraîner chez ces pères un stress susceptible de compromettre leur engagement envers l'enfant... Ces pères demeurent néanmoins très soucieux $d u$ contexte de pauvreté dans lequel ils vivent. Ils tentent de concilier les exigences de l'engagement envers leur enfant, qui apparaît prioritaire, et la précarité de l'emploi auquel ils peuvent aspirer étant donné leur faible niveau de scolarité : une tâche qui peut s'avérer complexe et ardue." des efforts consentis pour exercer leur rôle parental après la rupture en affrontant la pauvreté économique et sociale. Malgré leurs faibles revenus, ces pères font tout pour assurer le bien-être et la sécurité de leur enfant afin que celui-ci ne manque de rien, quitte à demander de l'aide de leur famille ou à avoir recours à des ressources dans la communauté. La pauvreté économique ne semble toutefois pas entraîner chez ces pères un stress susceptible de compromettre leur engagement envers l'enfant, tel que décrit par Elder et collab. (1985) et Fagan (2000). Il est possible que les participants à l'étude ayant adopté à l'égard de leur rôle paternel une vision plus large et moins traditionnelle, soient moins affectés par la pauvreté. Car, ils ne considèrent plus la dimension de pourvoyeur économique comme la composante centrale et unique de leur rôle parental. Ces pères demeurent néanmoins très soucieux du contexte de pauvreté dans lequel ils vivent. Ils tentent de concilier les exigences de l'engagement envers leur enfant, qui apparait prioritaire, et la précarité de l'emploi auquel ils peuvent aspirer étant donné leur faible niveau de scolarité : une tâche qui peut s'avérer complexe et ardue. Enfin, le fait de rester engagés après la rupture et de s'occuper seuls de leur enfant semble exposer ces pères à la surveillance et au jugement de l'entourage. Certains témoignent avec amertume de la méfiance et du discrédit dont ils sont l'objet dans l'exercice de leur rôle parental, et tout particulièrement pour les éléments qui ont trait à la relation avec l'enfant, comme les soins corporels et la discipline. L'analyse révèle que le maintien de l'engagement de ces pères séparés et pauvres, loin de s'appuyer sur des qualités extraordinaires, semble reposer sur des éléments "ordinaires ", mais non moins fondamentaux, dont certains rappellent des facteurs facilitant le maintien de l'engagement paternel (Gaudet et collab., 2005) et plus largement certains facteurs de protection de la résilience soit : a) des valeurs : plus particulièrement celles centrales accordées à l'enfant et au rôle de père; $b$ ) des perceptions : que les rôles parentaux ne sont pas liés au sexe, d'être un père compétent. Un sentiment qui évolue au cours du processus suivant la rupture et s'accroît au contact de l'enfant; c) des traits de personnalité : la capacité de compter sur soi-même pour changer les choses, jumelée à la capacité 
"Pour se représenter ce qu'est pour elles un père engagé après la rupture, les participantes traitent plusieurs informations, parfois contradictoires. Les croyances qu'elles entretiennent à l'égard des rôles parentaux s'ajoutent, voire se confrontent, à leurs expériences personnelles, aux renseignements issus de l'observation du père et de l'enfant depuis la séparation et, à la fois, au constat des bénéfices qu'elles retirent de l'implication du père." d'accepter de l'aide et d'en demander; d) des mécanismes de soutien ambiants : la présence constante d'un réseau de soutien et l'accès à des ressources dans la communauté.

\section{Le point de vue des mères}

L'influence maternelle est déterminante pour l'engagement paternel (Turcotte et collab., 2001) et la mère est souvent qualifiée de " garde-barrière " (gatekeeper) de la relation entre le père et l'enfant (De Luccie, 1995). Pourtant, peu de chercheurs ont étudié l'influence de la mère sur la continuité de l'engagement du père après la séparation conjugale, et plus rarement encore en contexte de pauvreté. En continuité avec la précédente, cette étude vise à explorer le phénomène du maintien de l'engagement paternel en donnant cette fois la parole aux mères.

Pour se représenter ce qu'est pour elles un père engagé après la rupture, les participantes traitent plusieurs informations, parfois contradictoires. Les croyances qu'elles entretiennent à l'égard des rôles parentaux s'ajoutent, voire se confrontent, à leurs expériences personnelles, aux renseignements issus de l'observation du père et de l'enfant depuis la séparation et, à la fois, au constat des bénéfices qu'elles retirent de l'implication du père.Au terme de ce processus, les mères interrogées disent qu'elles s'attendent du père engagé qu'il soit présent pour l'enfant, et qu'il lui offre, idéalement, une présence de qualité. Elles rejoignent en cela les travaux d'Amato et Gilbreth (1999) qui insistent sur le bénéfice pour l'enfant d'entretenir avec son père une relation affective de qualité après la rupture. Pour ces mères, le père engagé, c'est aussi le parent qui les soutient dans leur rôle de mère, c'est-à-dire celui qui est disponible, assume ses responsabilités parentales et leur est complémentaire. Les participantes, qui toutes vivent de l'aide sociale, ont par ailleurs peu abordé la dimension du soutien économique de leur ex-conjoint. Encourager ou freiner le maintien de l'engagement paternel de leur ex-conjoint? La plupart des participantes, malgré les critères de sélection de l'étude, se sont débattues pour savoir quelle orientation donner à leur influence avec des éléments aussi déterminants que contradictoires que : la valeur accordée au lien de l'enfant avec son père biologique; les avantages liés à 
"Malgré leur situation de précarité, une vision plutôt traditionnelle des rôles parentaux et la valeur indéniable qu'elles accordent au rôle de mère, la majorité d'entre elles reconnaissent l'importance de maintenir le lien entre l'enfant et son père biologique, même celles qui disent avoir vécu de la violence conjugale..." l'implication du père; la qualité de leur relation avec l'ex-conjoint; et pour certaines, les expériences de violence conjugale. Malgré leur situation de précarité, une vision plutôt traditionnelle des rôles parentaux et la valeur indéniable qu'elles accordent au rôle de mère, la majorité d'entre elles reconnaissent l'importance de maintenir le lien entre l'enfant et son père biologique, même celles qui disent avoir vécu de la violence conjugale (le tiers des participantes). Et loin de vouloir y faire obstacle, la plupart encouragent le maintien de l'engagement paternel. Leurs propos suggèrent que leur influence sur la continuité de l'engagement paternel s'exerce selon deux axes : dans leur relation avec l'enfant et dans la relation avec leur ex-conjoint. Dans leur relation avec leur enfant, la plupart disent user au quotidien de différentes stratégies, soit évoquer le père, le faire "exister ", se montrer ouvertes à lui parler, ou encore soutenir l'enfant dans l'attente de son père et lors des changements de résidence. Certaines insistent aussi pour que le père de l'enfant ne soit pas dénigré dans leur famille, ni par leurs proches. Leur influence à travers la relation avec l'ex-conjoint, le père de l'enfant, est dynamique et se transforme entre le début de la séparation et le moment de l'entrevue. Dès la décision de rompre, la majorité des mères interrogées disent s'être campées comme la principale responsable de leur enfant, convaincues qu'il est normal qu'elles contrôlent et régulent la place qu'occupera le père auprès de l'enfant, s'inscrivant ainsi dans la fonction de "garde-barrière ". Certaines expriment néanmoins avoir eu une certaine ambivalence : désirant rester le parent principal de l'enfant, en même temps qu'elles souhaitaient une relation parentale sur la base d'une collaboration qui, par ailleurs, les inquiétait. Plusieurs en sont venues néanmoins à encourager une présence plus active du père et à contribuer avec lui à la construction d'une alliance parentale, pour le bien-être de leur enfant. Si le contrôle exercé sur le père s'assouplit chez plusieurs mères devenues plus confiantes, il a tendance à demeurer très strict chez celles qui disent avoir vécu des expériences de violence conjugale. Contrairement à la représentation du père engagé des autres participantes, pour celles qui disent avoir vécu des expériences de violence conjugale, la simple présence du père dans la vie de l'enfant, peu importe 
la qualité ou la fréquence de cette présence, est déjà comprise comme une certaine forme d'engagement. Cependant, une telle représentation de l'implication du père après la rupture serait moins bénéfique à l'enfant que des interactions de qualité. Parmi les participantes qui rapportent des expériences de violence conjugale, de rares mères révèlent avoir eu à l'égard de leur enfant des comportements négligents. Comportements qui semblent incités soit par la peur de générer un conflit avec leur ex-conjoint qu'elles craignent ou encore par celle de perdre les avantages liés à sa présence. Ces résultats suggèrent d'approfondir la compréhension du phénomène de l'engagement paternel en contexte de violence conjugale, encore peu étudié (Sternberg, 1997), et réitèrent l'importance d'intervenir avec vigilance auprès de ces familles dont certaines restent vulnérables après la rupture.

Enfin, l'étude renforce la pertinence d'encourager et de soutenir la solidarité entre les parents, même après la rupture. Les conditions d'une telle alliance, énoncées par les participantes, sont susceptibles d'inspirer la pratique des intervenants œuvrant auprès des familles séparées. Les enfants, et tout particulièrement ceux d'âge préscolaire, en pleine période de développement, tirent de plus grands bénéfices si leurs parents, malgré la rupture et un contexte adverse, s'accordent sur les règles de vie, font équipe et leur offrent une présence de qualité.

\section{Ce que ces deux études nous enseignent}

Si plusieurs convergences se dégagent des propos des mères et des pères sur le maintien de l'engagement paternel, il y a par contre des thèmes qui ont été abordés de façon unilatérale dans une étude, sans trouver d'écho dans l'autre. 


\section{Convergences entre les deux points de vue des mères et des pères}

\section{Perception de la mère comme parent principal}

"La reconnaissance $d u$ rôle maternel semble s'imposer d'emblée, contrairement à celle du rôle du père. De fait, durant la période qui suit immédiatement la séparation, les mères interrogées autant que les pères décrivent la mère comme le parent principal. Et leurs propos indiquent qu'ils agissent comme tel, alimentant ainsi un premier repli vers la mère."
Pour les mères participant à l'étude, il allait de soi que l'enfant leur soit confié par la cour ou à la suite d'une entente à l'amiable. La reconnaissance du rôle maternel semble s'imposer d'emblée, contrairement à celle du rôle du père. De fait, durant la période qui suit immédiatement la séparation, les mères interrogées autant que les pères décrivent la mère comme le parent principal. Et leurs propos indiquent qu'ils agissent comme tel, alimentant ainsi un premier repli vers la mère. Rappelons que plusieurs pères ont révélé avoir vécu "un trou noir " après la rupture, certains ayant abusé alors d'alcool ou de drogues, d'autres ayant sombré dans un état dépressif. On comprend aisément qu'une telle situation n'est pas propice au développement d'une relation parentale envers leurs enfants, et que les mères, dans un tel contexte, aient été contraintes de protéger seules leurs enfants et d'en assumer la responsabilité.

\section{Valeur accordée à l'enfant}

Tous les pères et toutes les mères participant à l'étude placent l'enfant au centre de leur vie. Si la valeur que les mères accordent à leurs enfants semble acquise, il en va autrement pour les pères. En effet, les propos des pères interrogés témoignent du travail ardu nécessaire pour faire valoir la légitimité de leur rôle parental devant leurs ex-conjointes ou encore la justice. L'enfant représentait aussi une raison pour "sortir du trou noir ", y compris de l'abus d'alcool ou de drogues. Rappelons que la valeur centrale accordée à l'enfant par le père demeure un facteur favorisant le maintien de l'engagement paternel (Gaudet et collab., 2005).

\section{Valeur accordée au rôle de père}

Tous les pères interrogés étaient convaincus de l'importance de leur rôle et plusieurs d'entre eux ont dû le prouver, le défendre ou le négocier. De même, toutes les mères interrogées, même celles qui ont connu la violence conjugale, reconnaissent l'importance 
"Quant aux pères interrogés, au fur et à mesure qu'ils gagnent de l'assurance dans l'exercice de leur rôle parental, ils décrivent la nouvelle relation qu'ils ont développée avec leur enfant, laquelle semble regrouper, malgré la discontinuité, plusieurs critères de qualité..." de préserver le lien entre l'enfant et son père après la séparation. La valeur accordée au rôle de père est alimentée par leurs expériences personnelles, positives comme négatives, et par les besoins perçus ou clairement manifestés par leur enfant de voir son père. Cette valeur ne semble pas suffire pour qu'elles agissent en ce sens. Il y a plus. La plupart disent avoir eu besoin d'être rassurées sur la qualité de la nouvelle relation qui se développe, après la rupture, entre l'enfant et son père. Une fois confiantes, elles se montrent prêtes à la faciliter. Certaines défendront même devant leur famille et leur entourage l'importance du rôle du père auprès de leur enfant. Selon Gaudet et collab. (2005), l'intensité avec laquelle le père s'identifie au rôle parental est parmi les facteurs facilitant le maintien de l'engagement paternel les plus documentés. Pour ces mères, la valeur qu'elles attribuent au rôle paternel pour leur enfant semble toutefois devoir être associée à la confiance dans les habiletés parentales de leur ex-conjoint.

\section{Une relation de qualité entre l'enfant et son père}

Les mères, pour la plupart, s'attendent à une présence de qualité du père envers son enfant et lorsqu'elles sont rassurées, elles sont portées à lever peu à peu la mainmise sur «la barrière ». Quant aux pères interrogés, au fur et à mesure qu'ils gagnent de l'assurance dans l'exercice de leur rôle parental, ils décrivent la nouvelle relation qu'ils ont développée avec leur enfant, laquelle semble regrouper, malgré la discontinuité, plusieurs critères de qualité (Amato et Gilbreth, 1999). De fait, le sentiment de compétence des pères évolue au cours du processus suivant la rupture et s'accroît au contact de l'enfant. Des études rapportent que le sentiment des pères séparés d'être de bons parents (Hetherington et Stanley-Hagan, 2002) faciliterait le maintien de leur engament ainsi que la perception d'avoir du pouvoir sur les décisions relatives aux activités et au bien-être de ses enfants (Madden-Derdich et Leonard, 2000). En contexte de pauvreté, les enfants ont grand besoin de leur père dont l'engagement leur est bénéfique et les protège contre les mauvais traitements (Black, Dubowitz et Starr, 1997; Dubowitz, Black et Kerr, 2000) et contre les adversités liées à la pauvreté (Harris et Marmen, 1996). 
La relation entre les parents, ex-conjoints

Les participants des deux études relatent avoir éprouvé des sentiments d'ambivalence à l'égard de l'autre parent, avoir nourri des sentiments souvent contradictoires sur la place à lui donner, tiraillés qu'ils étaient entre le désir de s'affirmer, de se soustraire à son contrôle ou de s'en faire un allié ou une alliée. Les pères comme les mères ont rapporté avoir appris à construire une relation parentale avec l'ex-conjoint après la rupture. Plus la confiance s'installe et plus le couple parental s'inscrit dans une attitude qui s'apparente à la coparentalité. Les enfants, tout particulièrement ceux d'âge préscolaire, en pleine période de développement, bénéficient d'une alliance entre leurs parents après la rupture, surtout si ces derniers s'accordent sur les règles de vie, font équipe et leur offrent une présence de qualité. Une relation harmonieuse avec l'ex-conjointe, la mère des enfants, demeure un facteur reconnu propice au maintien de l'implication du père.

Si les propos des mères et des pères participant aux deux études se rejoignent sur plusieurs points facilitant le maintien de l'engagement paternel, d'autres sujets sont traités de façon singulière, sans écho dans l'autre étude.

\section{Propos de participants sans écho dans l'autre étude}

De l'étude auprès des mères qui considèrent leur ex-conjoint comme étant toujours engagé envers leur enfant a émergé un sujet préoccupant : celui de la violence conjugale que le tiers des participantes rapportent avoir expérimentée. Ces dernières relatent à quel point elles ont été déchirées entre le désir du père qu'elles voient grandir chez leur enfant et leur appréhension des effets néfastes potentiels de la présence du père, susceptibles de menacer la sécurité de leur enfant ou la leur. Rappelons que la précarité économique tout comme l'abus d'alcool et de drogues constituent des facteurs de risque de violence conjugale (Riou, Rinfret-Raynor et Cantin, 2003).

De leur côté, les pères ont abordé des dimensions généralement attribuées au père soit la discipline et le soutien financier; sujets 
qui, par ailleurs, n'ont pas été traités par les mères. L'exercice de la discipline représente pour la plupart des participants une source de tension. Alors que le père était traditionnellement l'investi de la position d'autorité dans la famille, celle de "préfet de discipline ", ce rôle, avec le temps, a été passablement dévalorisé, puis déchu dans les années 60 et 70 (Hurstel et Delaisi de Parseval, 1990). Malgré cela, plusieurs pères se sentent concernés par l'exercice de l'autorité et l'établissement de règles de discipline pour leurs enfants. Parmi les pères participant à l'étude, ceux qui manifestaient plus de difficultés avec la discipline voyaient leurs enfants à temps partiel ou peu souvent. Certains craignaient que la discipline fragilise davantage le lien déjà ténu avec leur enfant, ou d'autres avaient peur d'être soupçonnés alors de violence par leur ex-conjointe. Pour les pères interrogés, la discipline se révèle un sujet particulièrement sensible qui mériterait d'être exploré davantage. Car, l'autorité paternelle manifestée au quotidien dans la discipline et les jeux physiques permet à l'enfant, notamment à celui d'âge préscolaire, d'apprendre à contrôler ses émotions agressives (Paquette, 2004).

Malgré l'importance que représente pour l'enfant le soutien financier de son père après une rupture d'union (Amato et Gilbreth, 1999; Furstenberg, Morgan et Allison, 1987), ce sujet n'a pas été abordé par les mères interrogées qui vivaient toutes de l'assistanceemploi. Par contre, tous les pères ont parlé de leur précarité économique, s'inquiétant des conséquences néfastes à court et à long terme pour l'enfant et pour eux-mêmes. Selon Bouchard (2001), la situation des pères à faible revenu et occupant un emploi précaire est hautement vulnérable : il est nécessaire qu'ils soient soutenus concrètement. Parmi les pères interrogés, ceux qui ne pouvaient pas concilier les exigences familiales après la rupture et celles d'un travail précaire ont fait le choix d'être présents à l'enfant, du moins pour le moment. D'autres études comme celle de Lopez, Tremblay et Bergeron (à paraitre) rapportent des résultats similaires. 


\section{Des enjeux pour l'intervention clinique}

Pour éviter que des enfants de parents séparés, en plus d'être pauvres, soient aussi "pauvres " de père (Allard, Bourret et Tremblay, 2004), certains facteurs du maintien de l'engagement paternel après la rupture en milieu de pauvreté se dégagent. Ces facteurs s'interinfluencent constamment dans le processus. Nous les résumons sous forme de questions qui permettent d'évaluer la situation qui se présente à nous.

D'abord, le père doit désirer profondément s'impliquer auprès de son enfant malgré le contexte et les adversités. Il doit croire profondément en son rôle et en ses capacités de le remplir adéquatement. Comme l'avaient aussi identifié Ihinger-Tallman et collab. (1995), de même que Madden-Derdich et Leonard (2000), la représentation qu'il se fait de son rôle et la place qu'il donne à son identité de père vont être déterminantes dans son engagement paternel. Ce facteur interagit aussi de manière dynamique avec la croyance du père qu'il peut influencer la vie de son enfant. Ainsi, la première question à se poser est : le père veut-il s'engager auprès de son enfant?

En deuxième lieu, la mère doit faciliter cet engagement ou du moins ne pas le freiner. Pour ce faire, elle doit croire à l'importance pour l'enfant d'avoir un bon lien avec son père et elle doit bien distinguer entre la relation conjugale et la relation parentale. L'ouverture et la confiance de la mère se créent au fur et à mesure que prend forme la relation du père à l'enfant. À partir de l'observation et de l'évaluation qu'elle fait de la relation pèreenfant, elle module le contrôle qu'elle exerce sur la "barrière ". Certaines études montrent que des mères vivant en situation de pauvreté seraient enclines à bloquer l'accès du père incapable de contribuer financièrement aux besoins de l'enfant (Coley et Morris, 2002). Les mères plus confiantes tendent vers une alliance avec le père, une forme de coparentalité sans présence continue de l'autre parent et sans le lien conjugal qui existait avant la séparation. Plusieurs études le démontrent : la qualité de la relation entre les ex-conjoints est déterminante. Elle exerce un poids important 
"Un des défis de l'intervention demeure de sortir l'enfant de la triangulation dont il est souvent l'objet lors d'un divorce conflictuel pour amener les parents, à partir d'une analyse systémique, à constituer peu à peu une équipe parentale collaboratrice et fonctionnelle..." sur le niveau d'engagement qu'exerce le père (McBride et Rane, 1998; Turcotte et collab., 2001), particulièrement en contexte de séparation (De Luccie, 1995; Madden-Derdich et Leonard, 2000). La deuxième question peut être formulée ainsi : la mère veut-elle permettre au père d'exercer son rôle?

Troisièmement, les proches et les intervenants, doivent développer une perspective de coparentalité. Les parents n'exercent pas leurs rôles parentaux dans une île déserte. Le contexte dans lequel ils se trouvent influence fortement leurs perceptions tout comme il crée les conditions qui facilitent ou freinent l'engagement paternel. Notamment, le réseau social doit soutenir l'engagement paternel. Non seulement l'ex-conjointe joue un rôle important, mais aussi la famille et les amis du père. Il n'était pas rare que des hommes racontent que des membres de leur famille les ont poussés à se reprendre en main lors du «grand trou noir ", leur indiquant que leurs enfants avaient besoin d'eux. Les proches doivent aussi croire au rôle social du père (Allard et collab., 2004).

Un des défis de l'intervention demeure de sortir l'enfant de la triangulation dont il est souvent l'objet lors d'un divorce conflictuel pour amener les parents, à partir d'une analyse systémique, à constituer peu à peu une équipe parentale collaboratrice et fonctionnelle (Gonçalves et Grimaud de Vincenzi, 2003). Des pères et des mères de ces deux études disent avoir développé avec l'ex-conjoint une relation parentale positive, une forme d'alliance ou coparentalité pour le bien de leur enfant. Les conditions sur lesquelles repose une telle alliance dans un contexte adverse sont certes susceptibles d'inspirer la pratique des intervenants œuvrant auprès des mères et des pères séparés. Les résultats invitent donc à poursuivre la compréhension du fonctionnement des parents qui réussissent à faire équipe après la rupture conjugale. Les résultats suggèrent donc aussi de soutenir les mères et les pères vivant en situation de pauvreté à développer entre eux une solidarité parentale après la séparation, en portant une attention particulière aux parents de jeunes enfants. En effet, les enfants d'âge préscolaire, en pleine période de développement, ont grand besoin d'un encadrement chaleureux et constant de leurs deux parents. La 
"On ne peut valoriser l'engagement paternel et favoriser une coparentalité sans tenir compte $d u$ contexte familial. En fait, cette orientation générale exige d'être révisée lorsqu'on se retrouve en contexte de violence." question qui se pose dans l'intervention clinique pourrait être : comment aider les parents à établir des mécanismes de solidarité parentale pour le bien de leurs enfants? De fait, encourager la solidarité parentale, c'est reconnaître la continuité des responsabilités parentales de la mère et du père, par delà la séparation conjugale, ainsi que la valeur de leur lien respectif à l'enfant. C'est aussi prévenir le désengagement paternel.

Cependant, et quatrièmement, une certaine vigilance demeure de mise. On ne peut valoriser l'engagement paternel et favoriser une coparentalité sans tenir compte du contexte familial. En fait, cette orientation générale exige d'être révisée lorsqu'on se retrouve en contexte de violence. Lorsqu'il s'agit de violence du père à l'égard de son enfant, diverses mesures sont habituellement prises dans le cadre de la Loi sur la protection de la jeunesse. Cellesci peuvent aller d'une interdiction totale de contact à des visites limitées sous surveillance. Par ailleurs, les pratiques semblent plus floues lorsqu'il y a violence conjugale envers la mère des enfants sans interdiction de contact avec les enfants. Certains organismes privilégient une mesure plus radicale d'interdiction totale de contact des enfants avec le père dès qu'il $\mathrm{y}$ a violence conjugale. D'autres au contraire se centrent sur les besoins de l'enfant et favorisent le maintien du lien avec son père. Cependant, dans de tels cas, les répercussions sur la sécurité de la mère ne sont pas toujours clairement évaluées. Sans aucun doute, de nombreuses précautions doivent être prises dans de telles situations pour assurer à la fois la sécurité de l'enfant et celle de la mère. En fait, très peu de recherches sur le sujet ont été menées à ce jour. Beaucoup reste à faire pour bien comprendre les impacts sur l'enfant et aussi mieux définir les mécanismes pertinents à mettre en place dans de telles situations. D'où les questions suivantes : en contexte de violence, y a-t-il lieu de maintenir le lien avec le père? Si oui, quels sont les mécanismes à mettre en place pour assurer la sécurité de l'enfant et aussi celle de la mère? Dulac et collab. (2007) répondent à ces questions en disant que les enfants ont droit à leur père et les pères à leur enfant. Il revient alors, selon eux, à la justice et aux intervenants de mettre en place les conditions nécessaires pour assurer la sécurité de l'enfant et celle de l'ex-conjointe. 


\section{Conclusion}

"...les propos de ces pères et de ces mères en contexte de pauvreté se rejoignent sur les valeurs favorables au maintien de l'engagement paternel après la rupture. Des valeurs fondamentales qui peuvent être des leviers auprès des deux parents. "
Les stratégies déployées par ces pères et ces mères pour maintenir l'engagement paternel après la rupture peuvent inspirer la pratique auprès des familles en contexte de pauvreté. D'ailleurs, des intervenants se questionnent déjà sur la valeur accordée à l'engagement du père après la rupture et sur le soutien à lui apporter (Allard, 2001). On constate que plus d'intervenants se préoccupent du père. Pourtant, il semble que le courant dominant de l'intervention en matière familiale soit encore axé sur la mère (Dubeau,Turcotte et Coutu, 1999).Au sein de grands programmes destinés aux clientèles défavorisées, comme les services intégrés en périnatalité et petite enfance (SIPPE), l'intervention demeure généralement centrée sur la mère et l'enfant. Pour soutenir les pères, les intervenants ont peu d'outils répondant au modèle masculin orienté davantage sur les forces que sur les déficits. On constate qu'ils utilisent encore trop souvent avec les pères un modèle normatif conçu pour les mères (Gaudet et Devault, 2001).

Que retenir des propos des pères et des mères participant aux deux études? Leur analyse met en lumière leur convergence. En effet, les propos de ces pères et de ces mères en contexte de pauvreté se rejoignent sur les valeurs favorables au maintien de l'engagement paternel après la rupture. Des valeurs fondamentales qui peuvent être des leviers auprès des deux parents. Si le développement d'une alliance parentale est généralement bénéfique pour leur enfant et facilite le maintien de l'engagement du père, elle n'est pas une panacée pour tous les parents séparés. Beaucoup reste à faire pour mieux comprendre les impacts positifs et négatifs de l'engagement paternel dans un contexte de violence familiale ainsi que des mécanismes à mettre en place pour assurer la sécurité de toutes les personnes concernées. Par ailleurs, lorsque la situation familiale le permet, soutenir les deux parents séparés en contexte de pauvreté dans l'établissement d'une relation de qualité entre eux et avec l'enfant peut contribuer à réduire le nombre d'enfants qui, après la rupture de leurs parents, se retrouvent 
trop souvent doublement pauvres : pauvres économiquement et privés de père.

\section{Bibliographie}

ALLARD, Francine (2001). Comment faire de la place aux pères? Analyse des propos tenus dans les ateliers des intervenants des CLSC et du Centre-Jeunesse de la région de Québec 25 octobre 2001, document interne, Québec, Direction de santé publique de la Capitale-Nationale.

ALLARD, Francine, et Lise BINET (2002). Comment des pères en situation de pauvreté s'engagent-ils envers leur jeune enfant? Étude exploratoire qualitative, Québec, Régie régionale de la santé et des services sociaux de Québec, Direction de santé publique de la Capitale-Nationale.

ALLARD, Francine, Amélie BOURRET et Gilles TREMBLAY (2004). Rester engagé envers son enfant après la rupture du couple : Point de vue de pères vivant en contexte de pauvreté, Québec, Agence de développement de réseaux locaux de services de santé et de services sociaux de la CapitaleNationale, Direction de santé publique.

ALLARD, Francine, Amélie BOURRET et Gilles TREMBLAY (2005). Maintien de l'engagement paternel après la rupture : Point de vue de mères vivant en situation de pauvreté, Québec, Agence de développement de réseaux locaux de services de santé et de services sociaux de la CapitaleNationale, Direction de santé publique.

AMATO, Paul R., et Joan G. GILBRETH (1999). « Non resident fathers and children's well-being : A Meta-Analysis ", Journal of Marriage and the Family, No 61, p. 557-573.

AMATO, Paul R., et Juliana M. SOBOLEWSKI (2004). "The Effects of Divorce on Fathers and Children - Nonresidential Fathers and Stepfathers ", sous la direction de Lamb, M.E, The Role of the Father in Child Development, Fourth Edition, John Wiley et Sons, p.341-367.

BLACK, Maureen M., Howard DUBOWITZ et Raymond H. STARR (1997). "African American father in low-income, urban families : development behavior and home environment of their 3 year-old children ", Child Development, № 70, p. 967-978.

BOUCHARD, Camil (2001). «Le père développeur », sous la direction de Bolté, C., et collab., (dir.), Sur le terrain des pères. Projets de soutien et de valorisation du rôle paternel, Montréal, GRAVE.

COLEY, Rebekah Levine, et Jody Eileen MORRIS (2002). « Comparing father and mother reports of father involvement among low-income minority families ", Journal of Marriage and the Family, $\mathrm{N}^{\circ} 64$, p. 982-997.

DE LUCCIE, Mary F. (1995). « Mothers as gatekeepers : A model of maternal mediators of father involvement ", The Journal of Genetic Psychology, p. 115-131.

DUBEAU, Diane, Geneviève TURCOTTE et Sylvain COUTU (1999). "L'intégration des pères dans les pratiques d'intervention auprès des jeunes enfants et de leur famille », Revue canadienne de psycho-éducation, Vol.28, No 2, p. 265-278.

DUBOWITZ, Howard, Maureen M. BLACK et Mia A. KERR (2000). "Fathers and child neglect ", Archives of Pediatrics Adolescent Medicine, No 154, p. 135-141.

DUCHESNE, Louis (2006). La situation démographique au Québec, Québec, Institut de la statistique du Québec. 
DULAC, Germain (1995). "La rupture d'union et déconstruction du lien père-enfant ", Revue Prisme, Vol.5, N $\mathrm{N}^{\circ} 2-3$.

DULAC, Germain (1998). "Que nous disent les pères divorcés à propos des transitions familiales? » sous la direction de R. Dandurand, P. Lefebvre et J.P. Lamoureux, Quelle politique familiale à l'aube de l'an 2000?, Montréal, L'Harmattan.

DULAC, Germain, et collab. (2007). "L'expérience de pères séparés en médiation familiale ", Intervention, $\mathrm{N}^{\circ} 127$, p. 79-89.

ELDER, Glen H., Tri VAN NGUYEN et Avshalom CASPI (1985). «Linking family hardship to children's live ", Child Development, № 56, p. 361-375.

FAGAN, James (2000). "Head start fathers' daily hassles and involvement with their children ", Journal of Family Issues, Vol.21, № 3, p.329-346.

FURSTENBER G, Frank F., S. Philip MOR GAN et Paul D.ALLISON (1987). «Paternal participation and children's well-being after marital dissolution ", American Sociological Review, No 52, p. 695701.

FURSTENBERG, Frank F, et Christine W. NORD (1985). "Parenting apart : Patterns of child rearing after marital disruption ", Journal of Marriage and the Family, No 47, p. 893-904.

GAUDET, Judith, et Annie DEVAULT (2001). «Comment intervenir auprès des pères? : le point de vue des intervenants psychosociaux ", Intervention, $\mathrm{N}^{\circ} 114$.

GAUDET, Judith,Annie DEVAULT et Camil BOUCHARD (2005). «Le maintien de l'exercice du rôle paternel après une rupture conjugale : obstacles et facilitateurs, Revue canadienne de psychoéducation, Vol.34, Nº1, p. 21-40

GONÇALVES, Pedro, et Annie GRIMAUD DE VINCENZI (2003). «D’ennemis à coéquipiers : Le difficile apprentissage de la coparentalité après un divorce conflictuel», Thérapie familiale, Vol.24, No 3, p. 239-253.

HARRIS, Kathleen Mullan, et Jeremy K. MARMEN (1996). «Poverty, paternal involvement, and adolescent well-being ", Journal of Family Issues, Vol.17, № 5, p. 614-640.

HETHERINGTON, E. Mavis, et Margaret STANLEY-HAGAN (1986). «Divorced fathers : Stress, coping, and adjustment ", sous la direction de M.E. Lamb, The Father's Role Applied Perspectives, New York, Chichester, Brisbane, Toronto et Singapore, John Wiley et Sons.

HETHERINGTON, E. Mavis, et Margaret STANLEY-HAGAN (2002). "Parenting in divorced and remarried families ", sous la direction de M.H Bornstein, Handbook of Parenting, Being and Becoming a Parent, Mahwah (NJ) et Londres, Lawrence Erlbaum.

HUBERMAN, A. Michael, et Matthew B. MILES (1991). Analyse des données qualitatives. Recueil de nouvelles méthodes, De Boeck Université.

HURSTEL, Françoise, et Geneviève DELAISI DE PARSEVAL (1990). "Le pardessus du soupçon ", sous la direction de J.L. Delumeau, et D. Roche, Histoire des pères et de la paternité, Paris, Larousse.

IHINGER-TALLMAN, Marilyn, Kay PASLEY et Cheryl BUEHLER (1995). "Developing a middle-range theory of father involvement postdivorce », sous la direction de William Marsiglio, Fatherhood, Contemporary Theory, Research, and Social Policy, Thousand Oaks, Sage.

IINSTITUT NATIONAL DE SANTÉ PUBLIQUE DU QUÉBEC (2000). Le déploiement des programmes de type Naître égaux, Grandir en santé au Québec, Québec, ministère de la Santé et des Services sociaux. 
JOYAL, Renée, et collab. (2003). Le rôle des tribunaux dans la prise en charge des enfants après le divorce ou la séparation des parents. Québec, ministère de l'Emploi, de la Solidarité sociale et de la Famille.

KRURK, Edward (1993). Divorce and Disengagement-Patterns of Fatherhood within and beyond Marriage, Halifax, Fernwood.

LAMB, Michael E., Joseph H. PLECK et James A. LEVINE (1986). « Effects of increased paternal involvement on children in two-parent families ", sous la direction de R.A. Lewis et R.E. Salt, Men in families, Beverly Hills, Londres et Nouvelle-Delhi, Sage.

LE BOURDAIS, Céline, Heather JUBY et Nicole MARCIL-GRATTON (2000). Maintien des contacts pères-enfants après la séparation : le point de vue des hommes. Rapport de recherche soumis à l'Équipe sur les pensions alimentaires pour les enfants, Ottawa, ministère de la Justice du Canada.

LANOUE, Julie, et Richard CLOUTIER (1996). La spécificité du rôle du père auprès de l'enfant, Québec, Centre de recherche sur les services communautaires et Association des centres jeunesse.

LIEBOW, Elliot (1967). Tally's Corner. A Study of Negro Streetcorner Men, Boston et Toronto, Little, Brown and Co.

MADDEN-DERDICH, Debra A., et Stacie A. LEONARD (2000). "Parental role identity and fathers' involvement in coparental interaction after divorce : Fathers' perspectives ", Family Relations, Vol.49, №3, p. 311-318.

MARSIGLIO, William, et Mark COHAN (2000). "Contextualizing father involvement and paternal influence: Sociological and qualitative themes ", Marriage and Family Review, Vol.29, No 2/3, p. 75-95.

MCBRIDE, Brent A., et Thomas R. RANE (1998). "Parenting alliance as a predictor of father involvement : An exploratory study ", Family Relations, Vol. 47, p. 229-236.

MOSLEY, Jane, et Elizabeth THOMPSON (1995). "Fathering behavior and child outcomes : The role of the race and poverty ", sous la direction de William Marsiglio, Fatherhood and Contemporary Theory, Research, and Social Policy, Thousand Oaks, Sage.

PAQUETTE, Daniel (2004). "Le rôle du père dans la capacité du garçon à gérer son agressivité ", Revue de psycho-éducation, Vol.33, No 1, p. 61-73.

PROSPÈRE, Définition de l'engagement paternel, réf. du 10 janvier 2004, http://www.unites. uqam.ca/grave/prospere/pages/vision.htm.

QUÉNIART, Anne (2004). L'exercice de la paternité suite à la séparation conjugale : un parcours semé d'obstacles, Québec, Presses de l'Université Laval.

QUÉNIART, Anne, et François FOURNIER (1996). "Les pères "décrocheurs" : Au-delà des apparences et des discours ", Actes du 3e Symposium québécois de recherche sur la famille, Québec, Presses de l'Université du Québec.

RIOU, Ariane, Maryse RINFRET-RAYNOR et Solange CANTIN (2003). La violence envers les conjointes dans les couples québécois, Montréal, Institut de la statistique du Québec, http://www. stat.gouv.qc.ca/publications/sante/violence_femme98_pdf.htm

STERNBERG, Kathleen J. (1997). "Fathers, the missing parents in research on family violence ", sous la direction de Lamb, M.E., The role of the father in chid development, New York, Brisbane, Toronto et Singapore, John Wiley et sons. 
TURCOTTE, Geneviève, et collab. (2001). "Pourquoi certains pères sont-ils plus engagés que d'autres auprès de leurs enfants? Une revue des déterminants de l'engagement paternel ", Revue canadienne de psycho-éducation, Vol.30, No 1, p. 65-91. 